\title{
Lyme carditis presenting with an atypical rash
}

\author{
Dennys Franco-Avecilla MD, Cynthia Yeung BSc, Adrian Baranchuk MD
}

- Cite as: CMAJ 2020 May 25;192:E584. doi: 10.1503/cmaj.191660

See related articles at www.cmaj.ca/lookup/doi/10.1503/cmaj.191279 and www.cmaj.ca/lookup/doi/10.1503/cmaj.191194

A 56-year-old woman presented to the emergency department with a new well-defined erythematous patch on her right clavicular region (Figure 1), myalgia and chills. The patient was prescribed cephalexin for cellulitis by the emergency department physician; however, the patient returned 15 days later with palpitations and light-headedness. She remembered a possible tick bite while running outdoors in a region endemic for Lyme disease. Electrocardiography findings had evolved to first-degree atrioventricular (AV) block with a PR interval of $400 \mathrm{~ms}$ (Appendix 1, part A, available at www.cmaj.ca/lookup/suppl/doi:10.1503/cmaj.191660/-/ DC1). We admitted the patient to hospital for suspected Lyme carditis. She promptly received treatment with ceftriaxone (2 g daily) administered intravenously. We found no evidence of infiltrative disease or other important findings on subsequent echocardiography and cardiac magnetic resonance imaging.

Despite intravenous antibiotics, the patient's conduction abnormalities progressed to second-degree AV block by the fifth day of admission to hospital (Appendix 1, part B). However, after 9 days of treatment with intravenous antibiotics, electrocardiography findings had evolved to first-degree AV block (Appendix 1, part C) with 1:1 conduction on stress testing up to 164 beats/min (Appendix 1, part D). We changed the antibiotic to oral doxycycline (100 mg twice daily) for a 21-day total course of antibiotic therapy. Serology testing confirmed positive results for Lyme immunoglobulin (Ig)M/lgG enzyme immunoassay and Western blot. Electrocardiography conducted 6 weeks after discharge from hospital showed normal sinus rhythm and PR interval.

Lyme carditis occurs in 4\%-10\% of adults infected with Borrelia burgdorferi and presents as high-degree AV block in $90 \%$ of cases. Owing to the challenges of diagnosing Lyme carditis, a risk stratification tool has been developed. The Suspicious Index in Lyme Carditis (SILC) score evaluates the likelihood that a patient's highdegree AV block is caused by Lyme carditis, and this case would have been categorized as high likelihood. ${ }^{1}$ Although the traditional teaching on the erythema migrans rash has been the classic bull'seye (ring-within-a-ring) appearance, the literature has clearly described the uncharacteristic variants of erythema migrans in $25 \%-30 \%$ of cases, including lesions that are uniformly red, redblue or, occasionally, with a vesicular central region. ${ }^{2}$ Our patient's

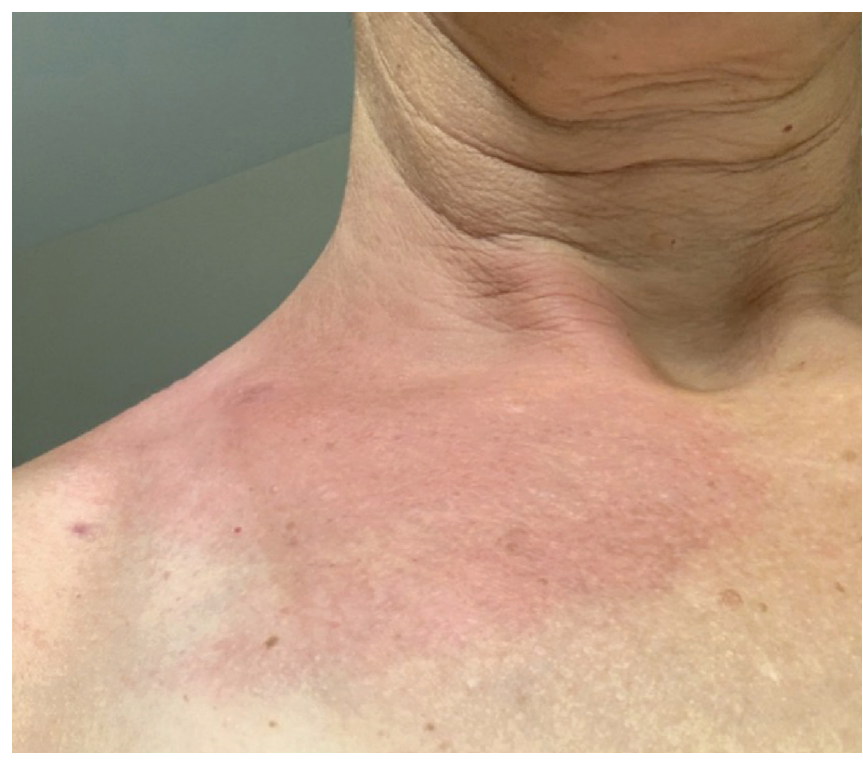

Figure 1: Atypical Lyme disease rash on the right clavicle of a 56-year-old woman with myalgia, chills and Lyme carditis.

well-defined rash was likely an atypical presentation of erythema migrans. A biopsy would have been conclusive; however, the rash had resolved by the time that Lyme carditis was suspected.

Given that most conduction abnormalities caused by Lyme carditis resolve with appropriate antibiotic therapy, recognition of atypical dermatologic presentations in the context of Lyme carditis prevents unnecessary permanent pacemaker implantation in these young and otherwise healthy individuals., ${ }^{3,4}$

\section{References}

1. Besant G, Wan D, Yeung C, et al. Suspicious index in Lyme carditis: systematic review and proposed new risk score. Clin Cardiol 2018;41:1611-6.

2. Schutzer SE, Berger BW, Krueger JG, et al. Atypical erythema migrans in patients with PCR-positive Lyme disease. Emerg Infect Dis 2013;19:815-7.

3. Wan D, Baranchuk A. Lyme carditis and atrioventricular block. CMAJ 2018;190:E622.

4. Yeung C, Baranchuk A. Diagnosis and treatment of Lyme carditis: JACC Review Topic of the Week [published erratum in J Am Coll Cardiol 2019;74:2709-11.] J Am Coll Cardiol 2019;73:717-26.

\section{Competing interests: None declared.}

This article has been peer reviewed.

The authors have obtained patient consent.
Affiliations: Alpha Medical Telemedicine Clinic (Franco-Avecilla), Toronto, Ont.; Department of Medicine (Yeung, Baranchuk), Queen's University, Kingston, Ont.
Correspondence to: Adrian Baranchuk, Adrian.Baranchuk@kingstonhsc.ca 